\section{Greece: The Hellenic Association of Medical Geneticists}

The Hellenic Association of Medical Geneticists (HAMG) was founded in 1982 by a group of scientists specializing in genetics. The founding members were 21 doctors, mainly pediatricians and biologists working as senior staff in genetic units of university departments and major hospitals in Greece. At present there are 160 active members of HAMG, and all medical specialties are represented, including biology, biochemistry, and dentistry; in 1988 the society had only 59 members.

The principal goals of HAMG are the establishment and promotion of genetics in Greece, research on genetic diseases, and dissemination of information to the public on available genetic services for the prevention of inherited disorders. Another basic objective is the scientific recognition and professional consolidation of geneticists in Greece.

The association's activities have focused on the development of a close collaboration and exchange of opinions among all medical doctors and other scientists who are involved in genetics in Greece. It is worth mentioning that the effort for close collaboration with other Balkan countries was fruitful and resulted in the establishment of a biannual Balkan Medical Genetics Congress. The first and the third Balkan medical congresses were held in 1994 and 1998 in Thessaloniki, Greece, with the participation of geneticists from Europe and the USA; the second meeting was held in Istanbul, Turkey; and the fourth was held in 2000 in NoviSad, Yugoslavia. During the third Balkan Congress in Thessaloniki, the establishment of a Balkan Medical Genetics Association was thoroughly discussed and decided upon.

Meeting seminars and conferences sponsored by HAMG have been held in Athens, Thessaloniki, Ioannina, Corfu, and Crete, with distinguished Greek and foreign lecturers.

As the only representative of the Greek geneticists, HAMG has applied to the Health Ministry for the foundation of the necessary Medical Genetic Centers, aiming at the improvement of genetic services in Greece. Also, following the universal demand for the establishment of a medical genetics specialty, HAMG has focused its activity in this direction. We hope that the recent establishment of medical genetics as an independent department of the University of Athens will promote these efforts.

One year ago, HAMG began publication of the first Greek journal on genetics; the Hellenic Journal of Human Genetics is intended not only for the members of HAMG, but also for every scientist interested in genetics.

Authors and members of the Hellenic Association of Medical Geneticists Christine Tsenghi, MD, President of HAMG Sophia Tzeli-Kitsiou, MD, Secretary General of HAMG 